Published in final edited form as:

Urol Oncol. 2020 February ; 38(2): 37.e21-37.e27. doi:10.1016/j.urolonc.2019.09.031.

\title{
Patterns of stereotactic body radiation therapy: the influence of lung cancer treatment on prostate cancer treatment
}

\author{
Bruce L. Jacobs, MD, MPH ${ }^{1,2}$, Jonathan G. Yabes, $\mathrm{PhD}^{2,3}$, Samia H. Lopa, $\mathrm{PhD}^{1}$, Dwight E. \\ Heron, MD, MBA, FACRO, FACR ${ }^{4}$, Chung-Chou H. Chang, $\mathrm{PhD}^{3,5}$, Justin E. Bekelman, \\ MD $^{6,7,8}$, Joel B. Nelson, MD ${ }^{1}$, Julie P.W. Bynum, MD, MPH ${ }^{9}$, Amber E. Barnato, MD, MPH, \\ MS $^{10,11}$, Jeremy M Kahn, MD, MSc ${ }^{12}$ \\ ${ }^{1}$ Department of Urology, University of Pittsburgh, Pittsburgh, PA \\ ${ }^{2}$ Center for Research on Health Care, University of Pittsburgh, Pittsburgh, PA \\ ${ }^{3}$ Division of General Internal Medicine, Department of Medicine, University of Pittsburgh, \\ Pittsburgh, PA \\ ${ }^{4}$ Department of Radiation Oncology-Hillman Cancer Center, University of Pittsburgh, Pittsburgh, \\ PA
}

${ }^{5}$ Department of Biostatistics, Graduate School of Public Health, University of Pittsburgh, Pittsburgh, PA

${ }^{6}$ Department of Radiation Oncology, University of Pennsylvania, Philadelphia, PA

${ }^{7}$ Division of General Internal Medicine, Abramson Cancer Center, University of Pennsylvania, Philadelphia, PA

${ }^{8}$ Leonard Davis Institute of Health Economics, University of Pennsylvania, Philadelphia, PA

${ }^{9}$ Department of Medicine, Division of Geriatric and Palliative Medicine, University of Michigan, Ann Arbor, MI

${ }^{10}$ Dartmouth Institute for Health Policy and Clinical Practice, Lebanon, NH

${ }^{11}$ Dartmouth Institute Geisel School of Medicine, Lebanon, NH

${ }^{12}$ Department of Critical Care Medicine, University of Pittsburgh, Pittsburgh, PA

\begin{abstract}
Introduction: Technology availability and prior experience with novel cancer treatments may partially drive their use. We sought to examine this issue in the context of stereotactic body
\end{abstract}

Correspondence Address: Bruce L. Jacobs, MD, MPH, Department of Urology, University of Pittsburgh, 5200 Centre Avenue, Suite 209, Pittsburgh, PA 15232, Phone: 412-605-3041. Fax: 412-605-3030. jacobsbl2@upmc.edu.

Publisher's Disclaimer: This is a PDF file of an unedited manuscript that has been accepted for publication. As a service to our customers we are providing this early version of the manuscript. The manuscript will undergo copyediting, typesetting, and review of the resulting proof before it is published in its final form. Please note that during the production process errors may be discovered which could affect the content, and all legal disclaimers that apply to the journal pertain.

Financial disclosures:

Amber Barnato is a former board member of the Society of Medical Decision Making 
radiation therapy (SBRT) by studying how its use for an established indication (lung cancer) impacts its use for an emerging indication (prostate cancer).

Methods: Using SEER-Medicare from 2007-2011, we developed prostate cancer-specific physician-hospital networks. Our primary dependent variable was SBRT use for prostate cancer and our primary independent variable was SBRT use for lung cancer, both at the network level. To assess the influence of SBRT availability and experiential use, we generated predicted probabilities of SBRT use for prostate cancer stratified by a network's use of lung cancer SBRT, adjusting for network characteristics. To assess intensity of use, we examined the correlation between the proportion of prostate cancer patients and lung cancer patients receiving SBRT within a network.

Results: We identified 316 networks that served 41,034 prostate cancer and 83,433 lung cancer patients. A network was significantly more likely to use SBRT for prostate cancer if that network used SBRT for lung cancer (e.g., in 2011, odds ratio [OR] 12.7; 95\% confidence interval [CI] 3.9-41.8). The Pearson's correlation between the proportion of prostate cancer patients and lung cancer patients receiving SBRT in a network was 0.34 , which was not statistically significant $(\mathrm{p}=0.12)$.

Conclusions: SBRT availability and experiential use for lung cancer influences its use for prostate cancer, but intensity of use for one does not relate to intensity of use for the other.

\section{Keywords}

prostate cancer; lung cancer; stereotactic body radiation therapy; physician-hospital networks; SEER-Medicare

\section{INTRODUCTION}

Health care costs are increasing dramatically, predominantly driven by the use of new technologies of uncertain effectiveness. ${ }^{1,2}$ Understanding this phenomenon is key to reducing health care spending growth by prioritizing the use of new technologies for highvalue care. One understudied driver of technology use in health care is the influence of availability and experiential use (i.e., the notion that having experience with a technology for one indication may influence its use for a second indication). ${ }^{3}$ As an example, robotic surgery was a new technology with similar outcomes to laparoscopy that demonstrated wide variation in use across multiple specialties. ${ }^{4}$ Its most rapid diffusion was in urology and gynecology — two related specialties thought to exhibit similar "cultural readiness" for using a new technology. ${ }^{4}$

We sought to examine the influence of technology availability and experiential use in the context of stereotactic body radiation therapy (SBRT) for prostate cancer. We chose to study SBRT for prostate cancer for several reasons. First, SBRT is a relatively novel treatment for prostate cancer introduced around 2007,5 which has a wide range of opinions regarding its optimal role. ${ }^{6,7}$ Second, unlike other recent prostate cancer technologies (e.g., robotic prostatectomy and proton-beam therapy) that are expensive, ${ }^{8}$ SBRT represents a potential cost savings due to its shorter treatment course, ${ }^{5}$ which could greatly impact prostate cancer care. Third, SBRT is an established treatment for lung cancer for which its use is more 
widespread, ${ }^{9}$ making it a valuable substrate for examining the influence of technology availability and experiential use.

We hypothesized that SBRT availability and experiential use, as defined by its use in lung cancer, would influence its uptake in prostate cancer. Further, we believed that the intensity of SBRT use for lung cancer would correlate with the intensity of SBRT use for prostate cancer.

\section{METHODS}

\section{Data Source and Study Population}

Using Surveillance, Epidemiology, and End Results (SEER)-Medicare, we performed a retrospective study from 2007-2011 where we empirically developed prostate cancerspecific physician-hospital networks and further classified these networks based on whether they also treated lung cancer patients. We defined physician-hospital networks based on prostate cancer patients because the primary outcome was the use of SBRT for prostate cancer among networks. To develop these networks, we identified men aged 66 years or older diagnosed with localized prostate cancer. We linked these data to the American Hospital Association (AHA) Annual Survey Database to obtain hospital characteristics. We excluded men who were 65 years old to ensure accurate comorbidity estimation. ${ }^{11} \mathrm{We}$ included fee-for-service beneficiaries eligible for both Medicare Parts A and B from 12 months prior until 12 months after diagnosis. We included men who had prostate cancer as their first and only cancer.

\section{Identifying physician-hospital networks}

We developed prostate cancer-specific physician-hospital networks based on previously designed methods (Appendix 1). ${ }^{12}$ Briefly, we assigned each patient to a prostate cancer treating physician based on the primary treatment received (i.e., active surveillance, surgery, brachytherapy, external beam radiotherapy, SBRT, proton-beam therapy, hormonal therapy, and cryotherapy). We then assigned each physician to a hospital based on where they most frequently practiced. If physicians practiced at multiple hospitals, we identified their primary hospital based on where they treated the greatest number of patients.

We only included networks anchored by hospitals located within SEER regions. We excluded networks with small numbers of prostate cancer patients (i.e., those with less than 30 patients overall and those without at least one patient in any given year) due to concerns for unstable estimates.

\section{Defining SBRT availability and experiential use}

We categorized physician-hospital networks into four mutually exclusive groups: those that used no SBRT, those that used SBRT for both prostate and lung cancer, those that used SBRT for prostate cancer only, and those that used SBRT for lung cancer only. We then examined the use of SBRT for lung cancer in a network (yes/no) as a proxy for SBRT availability and experiential use. We chose lung cancer because it represents the cancer most commonly treated with SBRT and because its use for lung cancer generally precedes that for 
prostate cancer. ${ }^{9,13}$ We excluded networks that contained less than 30 lung cancer patients and treated less than one lung cancer patient in a year. We also excluded networks where SBRT for prostate cancer preceded that for lung cancer because we were interested in how SBRT use for lung cancer influences its use in prostate cancer.

\section{Defining SBRT intensity}

To examine the intensity of SBRT use, we examined the correlation between the proportion of lung cancer patients in a network who received SBRT and the proportion of prostate cancer patients in that network who received SBRT.

\section{Variables}

We identified treatments using the Medicare Provider Analysis and Review, outpatient, and carrier files. We further identified patients who were treated with SBRT using Healthcare Common Procedure Coding System (HCPCS) codes. ${ }^{5,14}$ Teaching status was defined as membership in the Council of Teaching Hospitals, had an Accreditation Council for Graduate Medical Education approved residency program, or had a ratio of full-time equivalent interns and residents to beds of 0.25 or higher. ${ }^{15}$ Cancer programs were defined as those approved by the American College of Surgeons.

\section{Statistical Analyses}

We compared characteristics of the hospitals anchoring the networks using chi-square tests for categorical variables and ANOVA/Kruskal-Wallis tests for parametric/nonparametric continuous variables, respectively. Next, we examined the association of SBRT use for lung cancer with the use of SBRT for prostate cancer by fitting a network-level logistic regression model in which SBRT use for prostate cancer was the dependent variable and SBRT for lung cancer was the primary independent variable, adjusting for year and hospital characteristics (bed size, teaching status, and cancer program status). We tested for the interaction between SBRT use for lung cancer and year to determine whether the association of SBRT use for lung cancer and prostate cancer changed over time. We used robust standard errors to account for within physician-hospital network correlation over time. We performed sensitivity analyses where the primary independent variable was SBRT use for lung cancer one year prior and two years prior to SBRT use for prostate cancer.

Next, we performed analyses based on the networks that used SBRT for both lung and prostate cancer to examine whether there was a correlation between the intensity of SBRT use for lung cancer and the intensity of SBRT use for prostate cancer. We examined Pearson's correlation between the proportion of prostate cancer patients and the proportion of lung cancer patients in a network receiving SBRT. These proportions included the number of patients treated with SBRT in the numerator and the total number of treated patients in the denominator. Potential outliers and influential points were identified using standardized residuals and Cook's distance, respectively. We performed a sensitivity analysis where we included the networks that used SBRT for prostate cancer prior to lung cancer.

We performed all data management and analyses in SAS v9.4 (SAS Institute, Cary, NC) and R v3.5 (R Foundation for Statistical Computing, Vienna, Austria), respectively. All tests 
were two-sided and the probability of a type I error was set at 0.05 . The University of Pittsburgh institutional review board deemed this study exempt from full board review.

\section{RESULTS}

In refining the cohort, we excluded 301 networks representing 3078 patients affiliated with networks anchored by hospitals located outside SEER regions. We excluded 239 prostate cancer physician-hospital networks with small numbers of patients (i.e., those with $<30$ patients) and 2 networks without at least one patient in any given year. Similarly, we excluded 28 networks with less than 30 lung cancer patients. These exclusions represented $15.5 \%$ of the patient population. Our final cohort consisted of 316 physician-hospital networks, including 41,034 prostate cancer and 83,433 lung cancer patients.

Of the 316 networks, 150 (47.5\%) used no SBRT, 26 (8.2\%) used SBRT for both prostate and lung cancer, 4 (1.3\%) used SBRT for prostate cancer only, and 136 (43.0\%) used SBRT for lung cancer only (Table 1). Hospitals using SBRT for both prostate and lung cancer served the greatest number of patients, had more hospital beds, were affiliated with teaching, and were associated with American College of Surgeons-approved cancer programs (all $\mathrm{p}$ $<0.001)$.

The adjusted probability of networks using SBRT for prostate cancer stratified by SBRT use for lung cancer changed over time ( $\mathrm{p}=0.01$; generated from the interaction between SBRT use for lung cancer and year in a logistic regression model) (Figure 1). Within each year, physician-hospital networks that used SBRT for lung cancer were more likely to use SBRT for prostate cancer (demonstrated by odds ratios [OR] and $95 \%$ confidence intervals $[\mathrm{CI}]$ in Figure 1). As sensitivity analyses, we examined the adjusted probability of a network's use of SBRT for prostate cancer stratified by SBRT use for lung cancer one year and two years prior; in both cases, the findings were similar to the primary outcome (Appendix 2).

We examined the correlation between the proportion of prostate cancer and the proportion of lung cancer patients receiving SBRT among networks using SBRT (Figure 2). Two networks were excluded because they adopted SBRT for prostate cancer prior to lung cancer. The Pearson's correlation between the proportion of prostate cancer patients and lung cancer patients receiving SBRT in a network was 0.34 , which was not statistically significant $(\mathrm{p}=0.12) .{ }^{16}$ As a sensitivity analysis, we included the 2 networks that adopted SBRT for prostate cancer prior to lung cancer and found similar results (Appendix 3).

\section{DISCUSSION}

SBRT's use for lung cancer in a physician-hospital network is associated with the use of SBRT for prostate cancer. Although we cannot infer causation, only two networks used SBRT for prostate cancer prior to lung cancer and the findings were consistent when incorporating one- and two-year lags between the use of SBRT for lung cancer and the subsequent use of SBRT for prostate cancer, supporting the notion that SBRT for lung cancer influenced SBRT for prostate cancer. There was a weak but statistically insignificant correlation between the proportion of prostate cancer patients and the proportion of lung 
cancer patients in a network receiving SBRT, suggesting that the intensity of use for one cancer did not influence the intensity of use for the other.

In part, SBRT's availability is associated with its use due to compatibility, which is defined as the ability of a new technology to coexist with technologies and social patterns already in place, according to Rogers' Diffusion of Innovations model. ${ }^{3}$ Equipment used to deliver SBRT is expensive, costing roughly $\$ 2$ million. ${ }^{17}$ Especially for smaller centers, the expense of SBRT equipment may prohibit its adoption, reflected in its increased availability in larger hospitals, teaching hospitals, and among those with cancer programs. According to one survey, most non-SBRT users cite a lack of equipment as the reason for non-adoption. ${ }^{13} \mathrm{~A}$ network already delivering SBRT for lung cancer can offer it for prostate cancer without incurring additional capital expenditures. ${ }^{9,18}$

This phenomenon occurs with several other prostate cancer technologies. The daVinci Surgical System (Intuitive Surgical Inc., Sunnyvale, CA), which is used to perform robotic prostatectomies, costs over \$1 million and has high maintenance fees. ${ }^{19}$ However, hospitals can justify purchasing a robotic platform since it is used by several surgical specialties, including gynecology, general surgery, and thoracic surgery. ${ }^{20}$ Proton beam accelerators are another example, traditionally costing over $\$ 100$ million. ${ }^{8}$ Proton beam therapy's biggest benefit, arguably, is in treating tumors in confined spaces (e.g., brain and head and neck cancers) and in children due to the importance of precisely delivering radiation and avoiding adjacent normal tissue. ${ }^{21}$ However, these tumors are rare, and thus, proton centers rely on business from prostate cancer to fill unmet capacity. ${ }^{8}$

Experiential use may also help explain why SBRT use for lung cancer is associated with its use for prostate cancer. SBRT requires additional training not only for clinicians who must learn to devise the radiation plan, but also for other technicians (e.g., physicists, dosimetrists, therapists) who ensure that radiation is delivered safely and effectively. ${ }^{9}$ Having personnel at an institution who already deliver SBRT for lung cancer provides the infrastructure to help implement SBRT for other cancers. In addition, although clinicians delivering SBRT for lung cancer may not treat prostate cancer, their expertise may help colleagues trouble shoot when issues arise. These phenomena occur, in part, due to productivity spillovers whereby knowledge and skills are transferred to individuals in that environment. ${ }^{22}$ It is well known that physicians and staff gain experience learning from one another. ${ }^{22}$

The use of SBRT for prostate and lung cancer patients within networks is only weakly correlated, suggesting that intensity of use for one does not relate to the intensity of use for the other. In other words, high throughput of SBRT for lung cancer is not associated with high throughput of SBRT for prostate cancer.

Our findings have some important policy implications. By considering how SBRT's availability and experiential use impacts its use, policymakers and other stakeholders may improve the design of future interventions intended to either accelerate or decelerate its use, depending on the evidence. For example, networks encouraged by the evidence supporting SBRT for prostate cancer that use SBRT for other cancers could organize workshops and facilitating collaborations to improve training experience and expertise. This may become 
particularly relevant as the Center for Medicare and Medicaid Services considers implementing a mandatory bundled payment for radiation therapy across a wide range of cancers. $^{23}$

Our findings should be interpreted in the context of several limitations. First, defining SBRT's availability and experiential use solely by measuring its use in lung cancer is imperfect. SBRT is used in several cancers, including head and neck and liver cancer. ${ }^{9,21}$ However, lung cancer is the second most common noncutaneous cancer in men, occurring much more frequently than head and neck or liver cancer. ${ }^{24}$ Further, SBRT is used more in lung cancer than in any other cancer and became a viable treatment alternative for lung cancer long before it was an option for prostate cancer, making it a good choice for examining SBRT's availability for prostate cancer. ${ }^{9}$ Second, we are unable to infer causation from these analyses. However, several findings suggested that the correlation represented the influence of SBRT use for lung cancer on SBRT use for prostate cancer. In support of SBRT's earlier availability for lung cancer, 9,25 only two networks in our cohort used SBRT for prostate cancer prior to lung cancer. We also performed sensitivity analyses whereby we investigated one- and two-year time lags between the use of SBRT for lung cancer and subsequent use of SBRT for prostate cancer and the findings were similar. Third, since our primary outcome was at the hospital level, we omitted some patient and tumor characteristics from the analyses.

Despite these limitations, our study highlights some important findings. First, networks using SBRT for lung cancer were more likely to use it for prostate cancer. Second, the intensity of SBRT use for lung cancer did not influence the intensity of SBRT use for prostate cancer. Taken together, these findings highlight that we do not yet fully understand the drivers of SBRT use for prostate cancer, but that its use in lung cancer may play a role.

\title{
Supplementary Material
}

Refer to Web version on PubMed Central for supplementary material.

\section{Acknowledgments}

\author{
Funding:
}

Bruce Jacobs is supported in part by the GEMSSTAR award (R03AG048091), the University of Pittsburgh Physicians Academic Foundation, P30CA047904 from the National Cancer Institute, and the Henry L. Hillman Foundation.

Jonathan Yabes is supported in part by the by the University of Pittsburgh Clinical and Translational Science Institute-Research Education and Career Development Core (UL1 TR000005).

Julie Bynum is supported in part by an NIA grant (P01AG019783).

Amber Barnato is supported in part by the Levy Cluster in Health Care Delivery at Dartmouth.

Jeremy Kahn is supported in part by a career development award from the National Institutes of Health (K24HL133444). 


\section{REFERENCES}

1. Gawande A The cost conundrum. Available from URL: https://www.newyorker.com/magazine/ 2009/06/01/the-cost-conundrum [accessed February 20, 2018.

2. Newhouse JP. Medical care costs: how much welfare loss? J Econ Perspect. 1992;6: 3-21. [PubMed: 10128078]

3. Rogers E Diffusion of Innovations. 5th ed New York, NY: Free Press, 2003.

4. Juo YY, Mantha A, Abiri A, Lin A, Dutson E. Diffusion of robotic-assisted laparoscopic technology across specialties: a national study from 2008 to 2013. Surg Endosc. 2018;32: 1405-1413. [PubMed: 28842801]

5. Jacobs BL, Yabes JG, Lopa SH, et al. The early adoption of intensity-modulated radiotherapy and stereotactic body radiation treatment among older Medicare beneficiaries with prostate cancer. Cancer. 2017;123: 2945-2954. [PubMed: 28301689]

6. Yu JB, Cramer LD, Herrin J, Soulos PR, Potosky AL, Gross CP. Stereotactic body radiation therapy versus intensity-modulated radiation therapy for prostate cancer: comparison of toxicity. J Clin Oncol. 2014;32: 1195-1201. [PubMed: 24616315]

7. King CR, Freeman D, Kaplan I, et al. Stereotactic body radiotherapy for localized prostate cancer: pooled analysis from a multi-institutional consortium of prospective phase II trials. Radiother Oncol. 2013;109: 217-221. [PubMed: 24060175]

8. Pollack A Hospitals look to nuclear tool to fight cancer. Available from URL: https:// www.nytimes.com/2007/12/26/business/26proton.html [accessed December 2, 2018.

9. Pan HY, Jiang J, Shih YT, Smith BD. Adoption of Radiation Technology Among Privately Insured Nonelderly Patients With Cancer in the United States, 2008 to 2014: A Claims-Based Analysis. J Am Coll Radiol. 2017;14: 1027-1033.e1022. [PubMed: 28408078]

10. Altekruse SF, Kosary CL, Krapcho M, Neyman N, Aminou R, Waldron W, Ruhl J, Howlader N, Tatalovich Z, Cho H, Mariotto A, Eisner MP, Lewis DR, Cronin K, Chen HS, Feuer EJ, Stinchcomb DG, Edwards BK (eds.) SEER Cancer Statistics Review, 1975-2007, National Cancer Institute 2010.

11. Klabunde CN, Potosky AL, Legler JM, Warren JL. Development of a comorbidity index using physician claims data. J Clin Epidemiol. 2000;53: 1258-1267. [PubMed: 11146273]

12. Bynum JP, Bernal-Delgado E, Gottlieb D, Fisher E. Assigning ambulatory patients and their physicians to hospitals: a method for obtaining population-based provider performance measurements. Health Serv Res. 2007;42: 45-62. [PubMed: 17355581]

13. Pan H, Simpson DR, Mell LK, Mundt AJ, Lawson JD. A survey of stereotactic body radiotherapy use in the United States. Cancer. 2011;117: 4566-4572. [PubMed: 21412761]

14. Yu JB, Soulos PR, Cramer LD, Decker RH, Kim AW, Gross CP. Comparative effectiveness of surgery and radiosurgery for stage I non-small cell lung cancer. Cancer. 2015;121: 2341-2349. [PubMed: 25847699]

15. Trinh QD, Bianchi M, Sun M, et al. Discharge patterns after radical prostatectomy in the United States of America. Urol Oncol. 2013;31: 1022-1032. [PubMed: 22100070]

16. Taylor R Interpretation of the correlation coefficient: A basic review. Journal of diagnostic medical sonography. 1990;6: 35-39.

17. Azvolinsky A Early-stage lung cancer: trial compares radiosurgery to standard surgery. Available from URL: https://www.scribd.com/document/159249325/Lung-Article-Cyberknife-SBRT-forLung-Tumors-vs-Surgery [accessed January 14, 2018.

18. Pan HY, Haffty BG, Falit BP, et al. Supply and Demand for Radiation Oncology in the United States: Updated Projections for 2015 to 2025. Int J Radiat Oncol Biol Phys. 2016;96: 493-500. [PubMed: 27209499]

19. Lotan Y, Cadeddu JA, Gettman MT. The new economics of radical prostatectomy: cost comparison of open, laparoscopic and robot assisted techniques. J Urol. 2004;172: 1431-1435. [PubMed: 15371862]

20. Yu HY, Friedlander DF, Patel S, Hu JC. The current status of robotic oncologic surgery. CA Cancer J Clin. 2013;63: 45-56. [PubMed: 23161385] 
21. Brada M, Pijls-Johannesma M, De Ruysscher D. Proton therapy in clinical practice: current clinical evidence. J Clin Oncol. 2007;25: 965-970. [PubMed: 17350945]

22. Chandra A, Staiger DO. Productivity spillovers in healthcare: Evidence from the treatment of heart attacks J Polit Econ. 2007;115: 103-140. [PubMed: 18418468]

23. CMS proposes bundled payments for radiation oncology. Available from URL: https:// www.aimc.com/newsroom/cms-proposes-bundled-pavments-for-radiation-oncology [accessed August 23, 2019.

24. Siegel RL, Miller KD, Jemal A. Cancer Statistics, 2017. CA Cancer J Clin. 2017;67: 7-30. [PubMed: 28055103]

25. Timmerman R, Paulus R, Galvin J, et al. Stereotactic body radiation therapy for inoperable early stage lung cancer. JAMA. 2010;303: 1070-1076. [PubMed: 20233825] 


\section{Highlights}

- Availability and prior experience with treatments may partially drive their use

- $\quad$ SBRT availability for lung cancer is associated with its use for prostate cancer

- Intensity of SBRT for lung does not relate to intensity of use for prostate cancer 


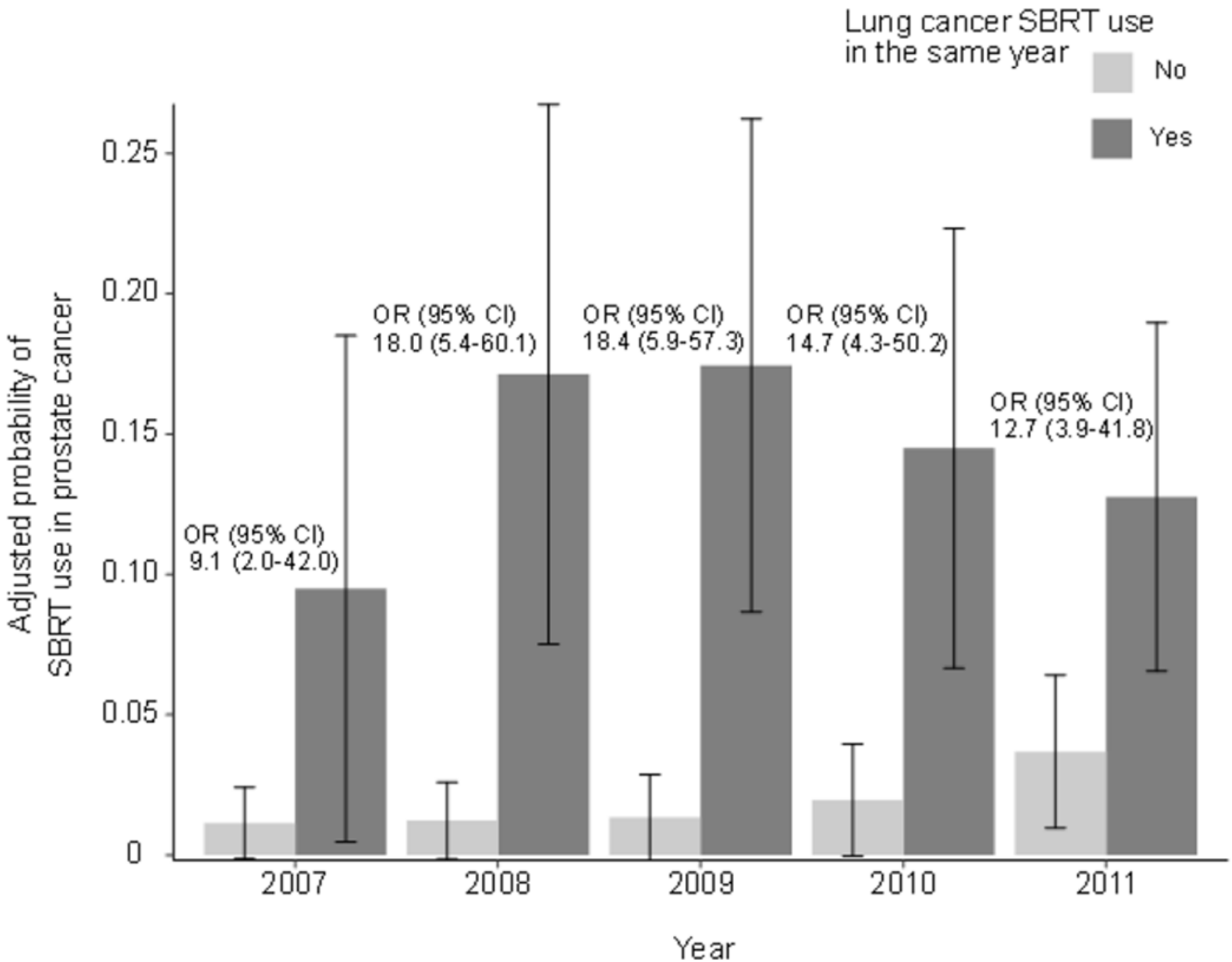

Figure 1. The adjusted* probability of networks using SBRT for prostate cancer stratified by SBRT use in lung cancer the same year.

Abbreviations: SBRT, stereotactic body radiation treatment

*Adjusted for hospital characteristics (i.e., bed size, teaching hospital, cancer program)

The effect of SBRT use in lung cancer on SBRT use in prostate cancer changes over time $(\mathrm{p}=0.01$; generated from the interaction between SBRT use for lung cancer and year in a logistic regression model). Robust standard errors were used to account for within physician-hospital network correlation over time. The error bars represent the 95\% confidence intervals (CI) for the estimated yearly proportions. The adjusted odds ratios (OR) and 95\% CI's for receiving SBRT for prostate cancer stratified by whether a network had SBRT for lung cancer are shown for each year. Within each year, physician-hospital networks that used SBRT for lung cancer were more likely to use SBRT for prostate cancer. 


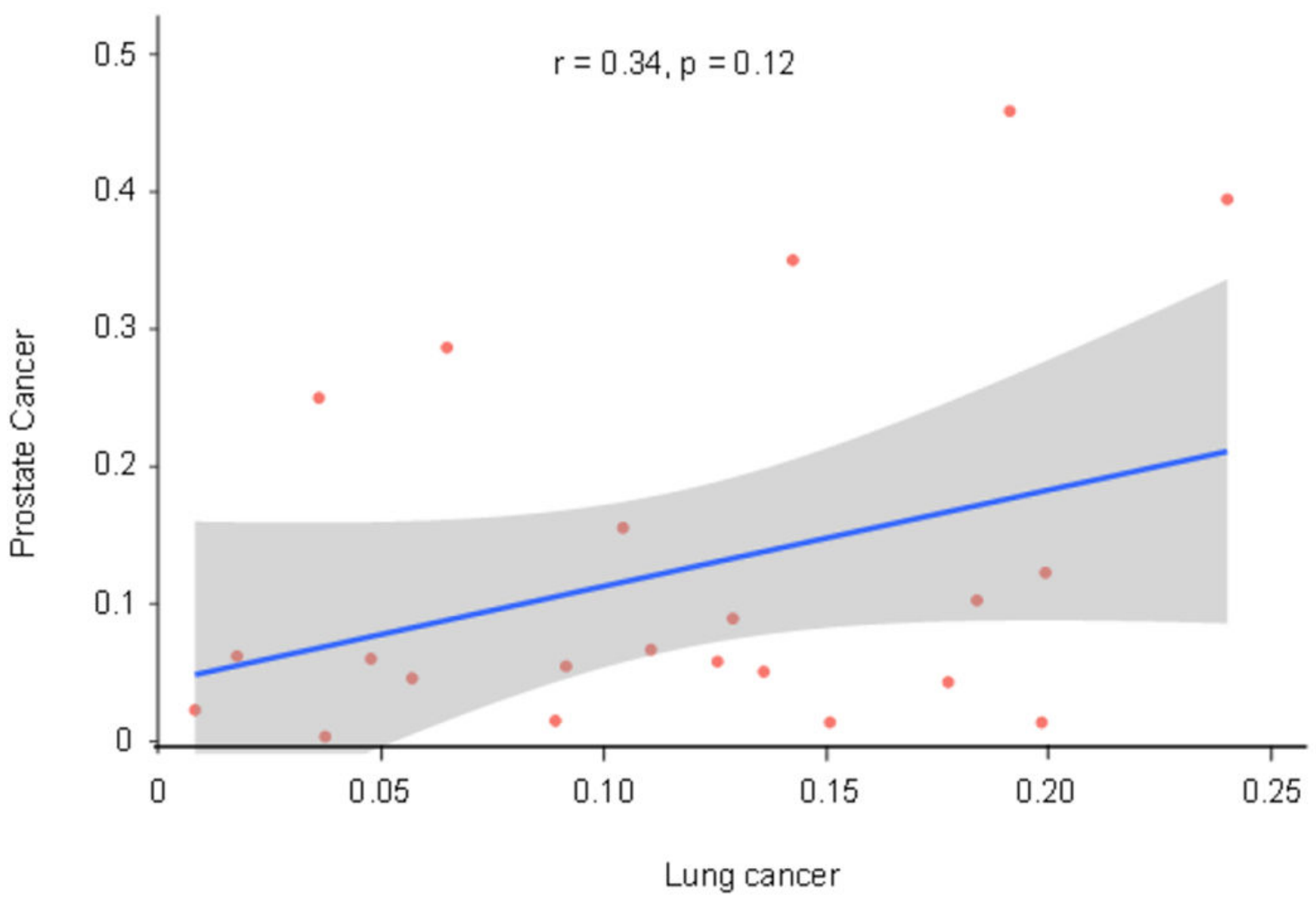

Figure 2. Correlation between the proportion of prostate cancer patients receiving SBRT in a network and the proportion of lung cancer patients receiving SBRT in that network

Abbreviations: SBRT, stereotactic body radiation treatment

Each dot represents one of the 22 physician-hospital networks that adopted SBRT for prostate cancer after its adoption for lung cancer. There is a weak but statistically insignificant correlation between the proportion of prostate cancer patients receiving SBRT in a network and the proportion of lung cancer patients receiving SBRT in that network $(\mathrm{r}=$ $0.34, \mathrm{p}=0.12$, Pearson correlation test). Gray area represents $95 \%$ confidence interval. 
Table 1.

Characteristics of the hospitals anchoring the physician-hospital networks

\begin{tabular}{|c|c|c|c|c|c|}
\hline \multirow[b]{2}{*}{ Hospital characteristics } & \multirow{2}{*}{$\begin{array}{l}\text { Networks not using } \\
\text { SBRT }(n=150)\end{array}$} & \multicolumn{3}{|c|}{ Networks using SBRT } & \multirow[b]{2}{*}{ P value } \\
\hline & & $\begin{array}{c}\text { Both prostate and } \\
\text { lung cancer }(n=26)\end{array}$ & $\begin{array}{l}\text { Prostate cancer } \\
\text { only }(n=4)\end{array}$ & $\begin{array}{l}\text { Lung cancer only } \\
(\mathbf{n}=136)\end{array}$ & \\
\hline \multicolumn{6}{|l|}{ Prostate Cancer } \\
\hline Total patients & 15,041 & 4500 & 324 & 21,169 & $<0.001$ \\
\hline Patients per network, mean (SD) & $100(82)$ & $173(119)$ & $81(39)$ & $156(127)$ & $<0.001$ \\
\hline $\begin{array}{l}\text { Patients per network, median } \\
\text { (IQR) }\end{array}$ & $78.5(50.2-125)$ & $126(80.5-280)$ & $84.0(60.5-104)$ & $120(76.8-181)$ & $<0.001$ \\
\hline \multicolumn{6}{|l|}{ Lung cancer } \\
\hline Total patients & 25,896 & 10,708 & 931 & 49,691 & $<0.001$ \\
\hline Patients per network, mean (SD) & $173(130)$ & $412(260)$ & $233(191)$ & $365(220)$ & $<0.001$ \\
\hline $\begin{array}{l}\text { Patients per network, median } \\
\text { (IQR) }\end{array}$ & $134(88.2-238)$ & $371(196-552)$ & $203(74.8-361)$ & $308(210-469)$ & $<0.001$ \\
\hline Bed size, $\mathbf{n}(\%)^{* *}$ & & & & & $<0.001$ \\
\hline 299 or less & $98(65.3)$ & $7(26.9)$ & -- & 47 (34.6) & \\
\hline 300 or more & $52(34.7)$ & $19(73.1)$ & -- & $89(65.4)$ & \\
\hline Teaching hospital, n (\%) & & & & & $<0.001$ \\
\hline No & $108(72.0)$ & $9(34.6)$ & $2(50.0)$ & $59(43.4)$ & \\
\hline Yes & $42(28.0)$ & $17(65.4)$ & $2(50.0)$ & $77(56.6)$ & \\
\hline $\begin{array}{l}\text { ACS approved Cancer program, } n \\
(\%)\end{array}$ & & & & & $<0.001$ \\
\hline No & $51(34.0)$ & $3(11.5)$ & $0(0.0)$ & $11(8.1)$ & \\
\hline Yes & $99(66.0)$ & $23(88.5)$ & $4(100.0)$ & 125 (91.9) & \\
\hline
\end{tabular}

Abbreviations: ACS, American College of Surgeons; IQR, interquartile range; SBRT, stereotactic body radiation treatment; SD, standard deviation

Percentages might not sum to 100 because of rounding

P values are for comparisons across all four groups using chi-square tests for categorical variables and ANOVA and Kruskal-Wallis tests for parametric and nonparametric data, respectively.

Some bed size data are suppressed due to small cell counts in compliance with SEER-Medicare guidelines 